\title{
LES MEDECINS JUIFS EN PROVENCE AU XVe SIECLE: PRATICIENS, NOTABLES ET LETTRES
}

\author{
Danièle lancu-Agou \\ Centre National de la Recherche Scientifique \\ Aix-en-Provence
}

Les médecins juifs apparaissent très largement dans les archives notariales de Provence. Dans le champ d'observation aixois, on peut les appréhender durant le XVe siècle, sous divers angles: en tant que praticiens zélés et fort prisés dans tous les milieux; en tant que notables de leurs collectivités, requis pour des fonctions et charges représentatives; en tant que lettrés soucieux d'acquérir ou échanger, léguer, recopier ou collectionner parchemins et manuscrits.

\section{DES PRATICIENS}

Malgré les interdits dont les médecins juifs sont frappés par la législation civile et ecclésiastique $\left({ }^{*}\right)$, il semble qu'ils aient été pleinement intégrés dans la société. En effet, la faveur qu'ils rencontraient auprès des grands de leur temps (tout autant qu'auprès des gens du commun) démontre combien les bulles papales et les objurgations ecclésiastiques demeuraient lettre morte, et combien leurs services étaient appréciés et en fait reconnus très officiellement.

II n'est qu'à citer les multiples exemples de praticiens juifs au service de gens d'Eglise pour s'en convaincre: à Aix, l'archevêque Avignon Nicolaï (mort 
en 1443) eut comme médecin, le juif Bonsenhor Vitalis, tandis qu'en 1450 , l'archevêque Robert Damiani avait à són service personnel le médecin juif Crescas Creyssent (on possède una lettre chaleureuse qu'il lui adresse in lui exprimant son voeu très vif de le voir embrasser le christianisme).

Les Comptes des Archevêques ou des Couvents font également état, tant à Aix qu'à Arles (ou Avignon), de pensions versées aux praticiens juifs: en 1423-24, dans les Comptes des Couvents des Dominicaines d'Aix, trois médecins juifs reçoivent pensions pour avoir soigné les religieuses (les mêmes Bonsenhor Vitalis, Crescas Creyssent cités supra, at Salomon Mordacays), tandis qu'à Arles, les Comptes de l'Archevêque mentionnent la pension qui fut versée à Mosse de Roquemartine qui avait soigné les familiers de l'évêque (dans le Comtat-Venaissin, durant tout un siècle, sur les quatre médecins qui soignèrent les Cordeliers d'Avignon, trois étaient juifs).

L'exemple du chirurgien Astruc de Sestier (qui laissait à sa mort en 1439 une si belle bibliothèque) est à cet égard significatif: quelques reconnaissances de dettes pour soins médicaux, petite intervention chirurgicale et médications diverses, permettent d'entrevoir ses démarches professionnelles à Aix au début du XVe siècle: assisté tour à tour de barbiers chrétiens, ou de médecins et chirurgiens juifs, il soigne en 1414 la blessure d'un laboureur d'Eguilles, en 1416 le fils d'un notaire aixois, tandis qu'en 1421 il traite les blessures d'un moine de l'Abbaye Saint-Victor de Marseille, et opère en 1435 un doigt de la main d'un laboureur aixois.

Ces divers actes cités à dessein reflètent la réalité quotidienne de ces temps reculés: la clientèle diversifiée d'un médecin juif, et les rapports de bon voisinage qui prévalaient malgré tout sur les règlementations édictées (il est à noter que le règlement des honoraires était subordonné au succès de l'intervention ou des soins donnés).

On peut parler aussi de bon voisinage à partir d'un autre exemple, une vente de recette médicale: deux frères, Josse Crescas de Riez et Mosse Crescas de Riez, fils d'un médecin juif originaire de Riez, se procurent chez un notaire, en 1481, à Aix où ils sont installés, un emplâtre ou «sinapisme», c'està-dire un cataplasme à base de moutarde.

Les praticiens juifs étaient enfin souvent requis pour les examens de constat de lèpre: les archives d'Aix rapportent le cas de ce médecin juif de SaintMaximin, Astruc Abraham, désigné en juin 1438, pour procéder à l'examen («palpatio») d'un patient chrétien de Tourves. A Arles, en 1428, deux «meges» chrétiens et le médecin juif Bendich Borrian signent le certificat de visite d'un lépreux. 
Mêmes occurences à Istres ou dans d'autres villes du Midi: on voit, pendant la peste, endémique à Avignon à la fin du XVe siècle, les chirurgiens juifs jouer un rôle notoire: ils sont nombreux à soigner les pestiférés, soit en ville, soit à l'hôpital Sainte-Marthe, tout en percevant certes des honoraires inférieurs à ceux de leurs confrères chrétiens.

\section{DES NOTABLES}

Au travers des écritures notariales, le perssonage du «magister medicus phisicus» (ou du «cirurgicus») juif apparaît beaucoup plus largement dans des agissements autres que professionnels.

Parvenant aisément à être représentatifs au sein et à l'extérieur de leur collectivité, ils sont très souvent at à tour de rôle «baylons ou syndics», c'est-àdire dirigeants communautaires.

A ce titre, ils collectaient l'impôt annuel qui pessait sur leur groupe (le fameuse «taille des Juifs» ou talhia judeorum) et se préoccupaient de sa répartition équitable. A ce titre aussi, ils étaient arbitres ou médiateurs dans les conflits qui agitaient les membres de leur communauté, et lorsque des différends s'élevaient pour des raisons fiscales ou autres, leur présence conciliatrice était toute requise.

Baylons, collecteurs d'impôts, médiateurs dans les conflits, les médecins juifs étaient également prêteurs et commerçants (à l'instar d'ailleurs de leurs homologues chrétiens, comme à Toulouse, où ils trafiquaient diverses marchandises, prêtant de l'argent et du blé). Ainsi l'aisance de ces praticiens n'étaitelle pas due uniquement aux seuls revenus que leur procurait l'exercice de leur art; marchands à leurs heures, ils s'adonnaient au crédit et au négoce, consentant des prêts d'argent ou de grains, spéculant sur l'élevage et vendant toutes sortes de produits, aussi bien du drap, de la laine, que des amandes (les Juifs en avaient le quasi-monopole à Aix) ou de la vaisselle vinaire.

Ils parvenaient ainsi à former un petit monde de nantis, aux larges assises matérielles, une strate mince et influente vivant dans l'aisance et dans la considération des gens de la contrée, coreligionnaires tout autant que Gentils.

Leurs réseaux familiaux, que l'on peut reconstituer grâce aux dots enregistrées chez notaires, sont caractéristiques: le plus souvent alliés aux familles de médecins, ils formaient de véritables dynasties médicales et n'hésitaient pas à chercher sur toute l'aire provençale (et comtadine) des conjoints de même 
rang social. C'est ainsi que seuls d'autres notables des juiveries proches ou avoisinnantes parvenaient à pénétrer ce réseau serré d'alliances médicales: les gros prêteurs et les dirigeants communautaires.

Le montant des dots assignées aux filles de médecins ou aux futures épouses de médecins (environ 500 florins en moyenne) est à cet égard éloquent et correspond aux practiques des couches supérieures de la société (600 florins est le montant moyen des dots des filles de nobles, de juristes et le montant minimal pour une fille de grand officier). On appréciera d'autant mieux un cas exceptionnel: la dot richissime de 2000 florins allouée par le médecin juif dracennois Massip Abram à sa fille Régine, lorsqu'il la marie à Aix en 1469 au futur médecin juif aixois Bonet Astrug de Lattes.

Ce faisceau d'éléments donne bien à penser que les médecins juifs formaient les personnalités les plus marquantes de la Juiverie, et certainement les plus cultivées.

\section{DES LETTRES}

En effet, pendant tout le XVe siècle, les registres notariés mentionnent les transactions qu'opèrent régulièrement les médecins juifs: des achats, des ventes et legs d'ouvrages qui n'étaient pas toujours médicaux.

Certes des volumes ayant trait spécifiquement à la médecine circulaient en grand nombre dans leur milieu, et c'est d'autant plus normal que la transmission du métier s'effectuait de père à fils, de beau-père à gendre, d'oncle à neveu, ce qui explique d'ailleurs la volonté d'endogamie médicale qui prévalait dans leur groupe; un groupe soucieux de préserver un héritage, de transmettre une science. C'est que l'on possède en définitive très peu de données sur leur formation de médecins, hormis quelques rares éléments, précieux, puisés à Arles pour le début du XVe siècle (un cas de licence obtenue par des étudiants juifs en médecine; une relation d'une soutenance de thèse par un candidat juif en présence de quatre médecins dont un chrétien; et enfin possibilité offerte à un autre étudiant de passer en mai 1459 l'examen de doctorat).

Hormis ces cas ponctuels trop rares l'apprentissage médical des médecins juifs reste quelque peu obscur, et c'est d'autant plus paradoxal que l'on sait la renommée le leur tradition professionnelle, acquise vraisemblablement «sur le tas», par la practique, dans le cadre (ou selon la richesse) de leurs bibliothèques: d'où l'importance capitale accordée en retour aux livres médi- 
caux, instruments recherchés, coûteux et indispensables à leur éducation et crédit scientifiques.

- On les lègue, tel le médecin juif d'Arles, Maître Bendich Borrian, qui dans son testament en 1441, laisse les quatre parties du fameux Canon d'Ibn Sina (Avicenne) à son beau-frère médecin Salamias Manelli.

- On.les fait recopier, et on engage des copistes à cet effet, comme cette «promesse d'écrire un livre de médecine en langue hébraïque» repérée dans un registre de notaire de l'année 1463. C'est d'ailleurs un futur médecin, Salomon de la Garde, qui commande ce travail et donne les fournitures: trente mains de papier et... six livres de chandelle!

- On les collectionne, tels ceux contenus dans la bibliothèque exemplaire du chirurgien Astruc de Sestier: nous les évoquerons plus loin, avec leurs auteurs et titres les plus significatifs.

D'autres manuscrits que ceux touchant à l'art de la médecine étaient également prisés et recherchés par cette «intelligentsia»: ceux qui traitaient autant de la Bible et du Talmud que de la philosophie si controversée des siècles précédents. Ces quelques exemples tirés de testaments ou de contrats matrimoniaux en témoignent: le médecin aixois Mordacays Salomon de Carcassonne dans son testament du 24 mai 1436, léguait à ses trois petits-fils trois Bibles hébraïques; son fils, un an plus atrd, acquerrait pour 60 flórins une biblia judaïca qui avait appartenu à un autre médecin juif de Trets, Dieulosal de Maneguet. En 1441, à Arles, Maitre Bendich Borrian, n'ayant pas eu d'enfants, se préoccupe de transmettre ses manuscrits aux parents, aux neveux de la prochaîne génération: à son beau-frère, il destine, outre Avicenne traduit en hébreu (cité supra), un traité talmudique sur «Les Semences» (Zera'im); et au neveu de sa femme, Maître Crescas Nathan (de la célèbre famille arlésienne des Nathan, érudits et lettrés connus), il lègue toute la Logique d'Aristote.

Les livres -objets de prix- figurent aussi en bonne place chez les médecins, dans les dots des nouvelles mariées: la Bible de 40 florins ajoutée aux 2000 florins dotaux alloués par Maître Massip Abram de Draguignan à sa fille Régine en 1469; ou alors «les livres tant hébreux que latins» (hélas non explicités) de Maître Abram Carcassonne, que sa veuve offre en dot à leur fille Stes lorsque celle-ci épouse le fils du médecin aixois Durand Gard en 1474 (la dot est évaluée à 500 florins comme suit: 300 en numéraires, et 200 «en vêtements, bijoux et livres tant hébreux que latins».

Mais l'exemple le plus saillant, celui qui mérite d'être développé, c'est incontestablement la bibliothèque exemplaire d'Astruc de Sestier, dont la liste 
de livres nous est rapportée grâce à l'inventaire après décès que fit dresser son fils Josse, également médecin, en 1439. Cette collection-modèle (179 ouvrages!) $\left({ }^{\star \star}\right)$-l'une des plus belles de l'historiographie juive de cette époque, véritable aubaine pour le chercheur - traduit les préoccupations intellectuelles étendues à tous les domaines (médical, scientifique, autant que traditionnel ou philosophique) d'un médecin juif de Provence, que rien ne situait hors du commun, mais qui s'est révélé à sa mort l'un des meilleurs bibliophiles de son temps.

\section{UN LETTRE-MODELE: MAITRE ASTRUC DE SESTIER, MEDECIN AIXOIS}

- En matiére médicale, les manuscrits (au nombre de 36) de Maître Astruc, chirurgien (on se souvient qu'il a soigné et opéré diverses blessures) attestent son goût pour la recherche chirurgicale et expérimentale. Ses rayonnages comportaient des manuels de chirurgie dont L'Abrégé sur la manière de traiter les plaies et les tumeurs de Gui de Chauliac, dont également Roland de Parmes, Lanfranc de Milan ou Théodoric, le chirurgien de Philippe le Bel. D'autres «classiques» étaient en sa possession: Razes en trois exemplaires dont l'Almansor; Avicenne en six volumes (dont quatre livres séparés du Canon et deux du Cantique de la Médecine ou Ardjuza); Isaac Israéli (deux Diététiques), ce médecin juif d'expression arabe, introduit et étudié à l'école de Salerne; la célèbre Maïmonide en un volume (le Traité sur l'asthme écrit en arabe et traduit en hébreu vers 1300), et Tesrif-al-Zahrawi (deux Théorica).

Outre ces manuels arabes ou juifs d'expression arabe, Astruc avait acquis les traductions hébraïques des Antidotaires (deux exemplaires de Nicolas Praepositus, 1150-1200); des Synonymes de Simon Gordo de Gênes, médecin de l'école de Salerne; du Lilium Medicinae de Bernard Gordon, professeur vers 1300 à l'école de médecine de Montpellier; de l'Introductorium Juvenum de Gérard de Solo qui traite des soins du corps, et enfin du Regimen Sanitatis d'Arnaud de Villeneuve.

Ainsi Maître Astruc, a-t-il été, dans sa discipline, largement pourvu et ouvert à la science de son temps qu'il avait pu assimiler grâce aux traductions hébraïques des oeuvres arabes, juives d'expression arabe, et latines. Ces traductions ont été pour la plupart le fait d'une couche zélée et diligente de Juifs venus d'Espagne (notamment les familles des Tibbon et des Kimhi) chassés d'Andalousie par les persécutions almohades, installés en Languedoc aux XIle siècle, et qui surent véhiculer en Occident grâce a leurs vertus linguistiques tout un savoir qui avait abondamment fleuri en terre ibérique. 
Notre chirurgien aixois - qui possédait aussi sept ouvrages d'astronomie et de mathématiques (dont deux d'Euclide) - réserva d'autre part un pan très large de sa bibliothèque à la science juive.

- Loi et Tradition juives. Presque la moitié de sa collection portait sur les disciplines traditionelles: dix volumes avaient trait à la Bible, dix-huit à l'exégèse biblique, dix-sept au Talmud avec sept commentaires de Rachi (le célèbre commentateur de Troyes), dix ouvrages de littérature rabbinique, sans compter trois exemplaires de lexicographie (dont deux Dictionnaires ou Shorashim de David Kimhi) et onze volumes de liturgie.

L'on ne peut ici livrer le détail de cet ensemble nourri (on l'a fait ailleurs. Cf. Bibliographie sommaire), mais il est à retenir l'intérêt évident que portait Maître Astruc pour le commentaire grammatical et exégétique du message biblique, et pour la littérature à caractère juridique et rituel.

Cette proportion importante d'ouvrages relatifs à la Loi et à la Tradition juives reflète ce qui fut au départ l'exclusif intérêt des communautés juives du Sud de la France (et d'Espagne) qui s'étaient adonnées à l'étude des disciplines rabbiniques et avaient produit d'éminents talmudistes.

Les curiosités philosophiques seront plus tardives et introduiront le délicat problème entre religion et science.

- Sciencies «profanes»: Philosophie (22 volumes): Maître Astruc possédait au début du XVe siècle les grandes oeuvres de la philosophie juive d'expression arabe (Maïmonide notamment, largement présent dans sa collection avec six volumes dont quatre Guides des Perplexes) qui, traduites par les Tibbonides, étaient devenues accessibles aux Provençaux, leur ouvrant des horizons nouveaux, ceux des sciences et de la philosophie. Leur vif engouement pour la philosophie, qui effaroucha les traditionnalistes, avait entraîné le controverse anti-maïmonidienne de 1230-1233.

On retrouve dans l'Inventaire de Maître Astruc les protagonistes de la deuxiéme lutte anti-philosophique qui se déclarera dans le conflit de 1303-1306: un Lévi ben Abraham de Villefranche de Conflent, bouc-émissaire de la nouvelle polémique, en deux volumes; ou Salomon ben Adret (un volume), qui dénonçait la confiance accordée à Aristote.

L'oeuvre d'Aristote semble d'allieurs avoir été parfaitement appréhendée par notre chirurgien aixois: il avait chez lui deux Physiques, une Ethique, un exemplaire des Météores et un volume Du Ciel et du Monde. Un traité d'Ibn Rosh (Averroës) garnissait aussi ses rayonnages, en même temps que d'autres 
livres de philosophie dont nous citons les plus célèbres: l'ouvrage L'Aiguillon pour les disciples de Jacob Anatoli, gendre de Samuel ibn Tibbon qui fut invité à la cour de Frédéric II à Palerme pour aider aux traductions de l'hébreu au latin ; un volume d'Ibn Falaquera Sur les divers degrés de la perfection intellectuelle; des livres d'Ibn Gabirol, d'Abou Nasar al-Farabi, ce dernier, philosophe du monde médiéval islamique dont l'influence fut considérable sur les philosophes juifs.

On aura pu juger, par cette diversité de volumes collectionnés, à quel point Maître Astruc eut une ouverture d'esprit sur les problèmes de son temps et sur les questions essentielles qui avaient précédé son temps. Son éclectisme et sa curiosité aux nouveautés annoncent le goût pour la bibliophilie, propre aux humanistes.

Ce praticien, comme ses homologues juifs provençaux, furent assurément les porteurs et agents de diffussion de la culture - tant biblique que talmudique, philosophique ou scientifique-dont ils avaient été les tenants privilégiés.

Pourtant, c'est chex ces médecins-lettrés que la cohésion religieuse est menacée aux générations suivantes: en effet, nous avons relevé une propension à la conversion dans les couches nanties du judaïsme provençal, chez les gros prêteurs, les baylons et invariablement chez les médecins. Etait-ce l'attrait exercé par la société environnante sur ces fils de notables? Toujours est-il que chez Maître Astruc qui a manifesté un tel souci pour la science juive, le fils, Moïse de Sestier, a très tôt rompu avec la tradition (il est devenu le nouveau-chrétien Guillaume Brici dès 1431); de même chez Maître Bendich Borrian d'Arles, un petit-neveu a embrassé la religion majoritaire, tandis que Maître Salomon de la Garde (qui se fit recopier en hébreu un livre de médecine) est repéré au début du XVle siècle sous le vocable d'Elzéar de Louis (ces nouveaux patronymes étaient empruntés comme c'etait l'usage aux familles chrétiennes qui parraînaient ces conversions). De tels exemples sont multiples: les deux filles du riche médecin Massip Abram de Draguignan ont rompu des mariages juifs pour se remarier, après conversion, à des conjoints chrétiens (dans les années 1470).

Etait-ce l'activité scientifique et philosophique de ces élites restreintes (hébraïsantes pourtant) qui menaçait inévitablement l'intégrité de leur judaïsme? Faut-il alors faire un lien entre aisance matérielle, milieu cultivé au fait des sciences dites «profanes» et abandon de l'attachement ancestral?. 
II y a là très certainement une tendance qui est symbolique, et qui n'est pas sans annoncer ou expliquer le large mouvement de conversion que l'on enregistrera plus tard, chez ces mêmes notables, au moment fatidique de l'expulsion des Juifs de Provence en 1500-1501. 
. 


\section{BIBLIOGRAPHIE SOMMAIRE}

Voir de l'auteur les études suivantes (où sont contenues toutes les indications bibliographiques requises):

IANCU-AGOU, Danièle, «L'inventaire de la bibliothèque et du mobilier d'un médecin juif d'Aixen-Provence au milieu du XVe siècle», Revue des Etudes Juives, tome CXXXIV (1-2), 1975, pp. $47-80$.

- Id., «Préocupations intellectuelles des médecins juifs au Moyen Age», Provence Historique, fasc. 103, 1976, pp. 21-44.

- Id., «Une strate mince et influente: les médecins juifs aixois à la fin du XVe siècle (14801500). Activités économiques et état social», Minorités, Techniques et Métiers, CNRS, Université de Provence, 1978, pp. 105-126.

- Id., "Documents sur les Juifs aixois et la médecine au XVe siècle» (Actes du 110e Congrès national des Sociétés Savantes, Montpellier, 1985), Santé, Médecine et Assistance au Moyen Age, Editions du C.T.H.S., Paris, 1987, pp. 251-262.

- Id., "Une vente de livres hébreux à Arles en 1434. Tableau de l'élite juive arlésienne», Revue des Etudes Juives, tome CXLVI (1-2), 1987, pp. 5-62.

Voir également les volumes collectifs Minorités, Techniques et Métiers (cité plus haut) et Le Corps Souffrant: Maladies et Médications, RAZO, n.4, Cahiers du Centre d'Etudes Médiévales de Nice, Université de Nice, 1984, avec notamment les articles de J. Shatzmiller, N. Coulet et L. Stouff.

Voir encore C. IANCU, "Les Juifs et la médecine à Montpellier au Moyen Age», La Médecine à Montpellier du Xlle au XXe siècle (sous la direction de L. Dulieu), Ed. Havas, 1988.

et L. DULIEU, «L'Ecole de Médecine de Montpellier a-t-elle été fondée par des médecins juifs», Les Juifs à Montpellier et dans le Languedoc du Moyen Age à nos jours (sous la direction de C. lancu), C.R.E.J.H., Université P. Valéry, Montpellier, 1988.

Ainsi que les travaux plus anciens de P. PANSIER, «Les médecins juifs à Avignon aux XIIle, XIVe et XVe siècles», Janus, 1910, pp. 421-451.

WICKERSHEIMER, E., Dictionnaire biographique des médecins français au Moyen Age, Paris, 1936, deux volumes. Deux suppléments ont été publiés par D. Jacquart, Paris, 1979 et 1981.

(*) Premier, semble-t-il en date, le concile de Béziers de 1246 interdit sous peine d'excommunication aux Chrétiens de faire appel à leurs soins, "car il vaut mieux mourir que devoir sa vie à un Juit».

Interdiction réitérée par les conciles d'Albi (1254), de Vienne (1267), par un avis de l'Université de Paris (1301), et par de nombreux conciles des XIVe et XVe siècles. 
Pour la Provence, la marginalisation prônée par les règlements de Charles II ou de Robert (interdiction aux patients chrétiens de solliciter les soins de praticiens juifs) connut bien évidemment des accomodements dans la vie quotidienne.

$\left.{ }^{* * *}\right)$ A titre de comparaison, il n'est pas indifférent de relever la dimension des bibliothèques chrétiennes aixoises contemporaines: 147 titres chez Jean de Vitrolles, 78 chez le maîtrerational Antoine Suavis, 157 chez le chancelier Jean Martin. 